\title{
Publish-and-Flourish: Using Blockchain Platform to Enable Cooperative Scholarly Communication
}

\author{
Emilija Stojmenova Duh ${ }^{1,2}$, Andrej Duh ${ }^{1}$, Uroš Droftina ${ }^{1}$, Tim Kos ${ }^{1,3}$, Urban Duh ${ }^{1}$, \\ Tanja Simonič Korošak ${ }^{4}$ and Dean Korošak $5,6, *$ iD \\ 1 Infinitcodex, Ltd., Maribor SI-2000, Slovenia; Emilija.Stojmenova@ltfe.org (E.S.D.); \\ andrej.duh@infinitcodex.com (A.D.); u.droftina@gmail.com (U.D.); \\ tim.kos@infinitcodex.com (T.K.); urban.duh@infinitcodex.com (U.D.) \\ 2 Faculty of Electrical Engineering, University of Ljubljana, Ljubljana SI-1000, Slovenia \\ 3 Institute of Mathematics, Physics and Mechanics, Ljubljana SI-1000, Slovenia \\ 4 TSK Studio, Maribor SI-2000, Slovenia; simonic.tanja@gmail.com \\ 5 Institute for Physiology, Faculty of Medicine, University of Maribor, Maribor SI-2000, Slovenia \\ 6 Faculty of Civil Engineering, Transportation Engineering and Architecture, University of Maribor, \\ Maribor SI-2000, Slovenia \\ * Correspondence: dean.korosak@um.si; Tel.: +386-2-2294-323
}

Received: 17 November 2018; Accepted: 24 April 2019; Published: 5 May 2019

\begin{abstract}
Scholarly communication is today immersed in publish-or-perish culture that propels non-cooperative behavior in the sense of strategic games played by researchers. Here we introduce and describe a blockchain based platform for decentralized scholarly communication. The design of the platform rests on community driven publishing reviewing processes and implements cryptoeconomic incentives that promote cooperative user behavior. The key to achieve cooperation in blockchain based scholarly communication is to transform today's static research paper into a modifiable research paper under continuous peer review process. We introduce and discuss the implementation of a modifiable research paper as a smart contract on the blockchain.
\end{abstract}

Keywords: scholarly communication; open science; blockchain; decentralisation; smart contract; continuous peer review; publish-and-flourish

\section{Introduction}

The Internet and social media in particular are the enablers of media convergence, a phenomenon characterized by flow of content and migration of users, linking together content, communication and computation [1,2]. However, besides positive effects of convergence such as the rise of collective processes of information consumption and consumer-generated media, we are recently witnessing the emergence of fast traveling fake news influencing collective human decisions [3,4], helped also by spreading of automated content generation [5]. Even reporting on results of scientific discoveries in traditional academic journals is not immune to overhyped or even fake claims that cause irreproducibility and distrust [6].

At first, fast migration and automation of content, and trust seem orthogonal to each other. However, the application of blockchain technologies [7] hold a promise to profoundly change and decentralize scholarly communication [8,9], bringing together spreadability, migration credibility and trust, based on open science principles [10]. Blockchains, in short, are digital ledgers of cryptographically validated transactions distributed in a network of nodes that validate each new block of transactions through some consensus mechanism before that block is immutably appended to the ledger [11-14]. Blockchains are architecturally and politically decentralized: there is no single point of failure in the infrastructure and there is no single governing body of a blockchain. All nodes in 
the blockchain network must, however, with a consensus agree on one state of a blockchain, therefore making blockchain a logically centralized system [12].

Like blockchain, science is architecturally (research infrastructures do not have a single point of failure) and politically (research is not governed by a single scientific authority) decentralized [15]. Science is, however, logically centralized (the scientific community must agree on one state-scientific truth) through "a process that does lead to a broadly shared consensus. It is arguably the only social process that does" [16]. In science, this consensus is reached through scholarly communication. Though "The goal of science, moreover, is to achieve the maximum degree of consensuality" [17], we see the consensus in science as a temporal, dynamic process that also influences peer review and its outcomes [18]. There are variations in the level of consensus and different approaches to confirmation [19] and reproducibility [20] across scientific disciplines. We find the broadly shared consensus at the core knowledge, while at the research frontier [21] there may simultaneously exist several (many) research consensuses.

Building of a truly decentralized and trusted scholarly communication model "prompts the articulation of the functions that academic publishing provides and how, if these are still required, they might be provided in decentralized models" [14].

Academic publishing (in print and electronic form) is a prevailing medium of scholarly communication that in the current form suffers from inefficiencies including slow, incomplete, inaccurate and unmodifiable communication [22].

Today, there exist three high-level models of academic publishing: paywalled (peer reviewed, free or fee for authors, readers pay to access the papers), open access (peer reviewed, fee for authors to publish, free access to published papers), and preprint repository (self-archiving or centralized archives of preprints, no fees, but no peer-review either). Scholarly publishers provide, from an economic point of view, rivalrous or non-rivalrous goods (research papers in the form of physical or digital texts) that can be excludable or non-excludable (behind paywall or free to access). However, a more careful analysis shows that a journal, as an instance of scholarly communication, is better understood as a knowledge club [23] where members of the community both produce and consume knowledge under voluntary and non-anonymously crowded participation, with exclusive access [23]. If scholarly publishing "works best as a club good" [23] and is "better understood as a social production technology" [24] then any innovative technological solution to scholarly communication problems must primarily focus on providing support for communities and their governance structure. Blockchain technologies are promising in this respect since at the core they are peer-to-peer networks governed by consensus mechanisms. Yet, despite technological advances in communication and publishing technologies that can provide efficient infrastructure governed by researchers [25], the journal structure of scholarly communication is still prevalent. We agree that "scholarly publishers need to shift their attention from products to communities; from journals to knowledge clubs" [24].

Consolidation of the academic publishing industry resulted in a market with limited competition and with ever increasing profits for a few large players [26-28]. Large profits are possible because the marginal costs of electronic publishing have no lower boundary (practically zero), and because paywalled or open access academic publishing stands on non-compensated work of researchers in three-fold roles: authors, reviewers and editors. Academic publishing was described as a coordination game (Bergstrom 2001) where users (authors, reviewers and editors) coordinate at journals and are stuck in one of the equilibria where they "continue to pay huge rents to owners of commercial journals" [29].

Large publishing companies have positioned themselves as centralized authorities, as gatekeepers of the quality of published research and providers of reputation for authors whose academic careers in turn depend on the products these same publishers provide. Academic life is immersed in publish-or-perish culture, the growing pressure on researchers to keep rapidly publishing scholarly content to survive in academia, resulting in fast-growing volume of published papers $[30,31]$. 
Such accelerated growth of published scholarly content slows down the progress in large scientific fields [32] and affects science trustworthiness when the quantity of published work is used as dominant metric for evaluation of researchers [33]. Trustworthiness in science is further eroded by arrival of predatory journals [34] the existence of which "is only the most pathetic facet of a much deeper cultural problem within science- a form of comic relief on the backdrop of a tragedy, which we should all take as a reminder of how far from our goals we have strayed" [35].

At the core of scholarly communication is peer review, a recommendation process underlying publication decisions that should guard the trust and guarantee high quality of published work. However, under publish-or-perish culture, peer review became flawed, non-transparent, inconsistent and biased [36-38]. The current situation in the academic publishing market where the scientific journals operate without the transparency of peer review process [39-41] is harmful to scientific progress [42-44], limits the participation of researchers from developing economies, is biased against young researchers, and creates wrong incentives for authors [45-48].

Are there ways out of the current scholarly communication paradigm? In terms of a coordination game, can the community of researchers move from the current publish-or-perish equilibrium into publish-and-flourish equilibrium?

Suggestions to change the current paradigm include morphing the self-archiving preprint repositories into the arXivs of the future [49], using disruptive technologies such as peer-to-peer applications changing distributions of financial resources between researchers and institutions [50], and decentralized blockchain solutions $[8,9,14,51]$ by creating platforms for open science [10] on the blockchain.

Following the analysis of inefficiencies of today's scholarly communication, a six-stage process approach of modifying the current system into an open science platform was proposed [22] with the implementation of open, continuous peer review as the last stage of the transformation process. Key to the shift from publish-and-perish to publish-and-flourish academic culture is a profound change in incentives for authors: “... the scientist's ultimate objective is no longer to get published, because everything is published. The objective is to influence future ideas and investigations, that is, what should be the key incentive in the first place" [22].

Here, we suggest that for such a shift a radical change of the concept of research paper itself is necessary - a change from unmodifiable to modifiable published scholarly content that by definition demands open and continuous review process. We show that distributed ledger technology (i.e., blockchain technology) based platform solution tailored to support open scholarly communication provides users (teachers, researchers, students) with mechanisms for building trust and reputation, protects privacy and operates with transparency and access through a decentralized peer-to-peer network. In particular, we propose a design for a platform that supports the implementation of encoding scholarly content into smart contracts-a code that runs on a blockchain. Such encoding allows the content to function as a computable autonomous entity that can interact with other computable autonomous entities on a blockchain and with human users on the platform.

\section{Theoretical Motivations}

An interesting set of experiments [52] explored how blockchain technology as a new medium could change storytelling by considering stories as currency-in this case as cryptocurrency, and noticed that both competitive and cooperative modes of user actions are induced by the distributed organisation of the blockchain. This opens up an intriguing possibility to explore the role of blockchain technology in strategic games [53] and in resolving social dilemmas occurring in scholarly communication set as game theoretical problems [54-59].

Let us consider two core processes in scholarly communication: publishing (making information available to public) and peer reviewing (recommendation process to publish/reject a paper) as games played by rational agents acting in both roles: as authors and as reviewers. 
The quality of published works and therefore trustworthiness in science critically depends on the social norms of peer reviewing process [56]. Diligent, responsible and honest peer reviews constitute a valuable resource of the scientific community-the scientific commons [60]. Under publish-or-perish pressure a researcher will prioritise her/his own publication output over providing quality reviews of other researchers' work, getting the benefit $B>0$ (obtaining a review from the commons) without the effort (cost) $e>0$ (contributing reviews to the commons). Each researcher can then play a strategy $C$ (cooperate), contributing reviews with the pay-off $B-e$ or can play a strategy $D$ (defect) with the pay-off $B$ if enough other researchers (for instance more than $N$ ) in the community cooperate. If there are not enough cooperators in the community, the pay-off of a cooperator is $e$, and the pay-off of a defector 0 . The game structure is given in Table 1.

Table 1. The tragedy of the commons game structure.

\begin{tabular}{ccc}
\hline & $\# C>N$ & $\# C<N$ \\
\hline$C$ & $B-e$ & $-e$ \\
$D$ & $B$ & 0 \\
\hline
\end{tabular}

With the pay-off structure: $B>B-e>e>0$, a rational, strategic choice is to play $D$ with the pay-off 0 , thus exhausting the commons and causing "the tragedy of the commons" [61,62] in this case the scientific commons.

Social mechanisms like indirect reciprocity [63] built on reputation of community members were shown to be able to resolve the commons dilemma, sustain cooperation (and thus preserve the commons) in certain repeated games and help overcome the "tragedy of the commons" [64]. More generally, for a successful resolution of social dilemma the interventions in strategies should focus on four key parts: information, identity, institutions and incentives [65].

The key principles of blockchain technology as enabler of decentralized scholarly communication are perfectly aligned with these foci of interventions and their core motives: understanding, belonging, trusting and self-enhancing [65]. Blockchain, a distributed immutable database with equal and full transparency of information to all users without centralized authority, reduces uncertainty and increases understanding. Transactions based on pseudonymous peer-to-peer communication, with a choice for a user to opt for proof of identity to others, induces a strong sense of community with mutual trust between communicating parties in contrast to groups with completely anonymous communication where it is hard to imagine that consistent and reliable reputation systems might emerge. The questions of anonymity, pseudonimity or disclosed identity in blockchain technologies are currently important legal and regulatory issues related to General Data Protection Regulation (GDPR) [66].

Integrity of the blockchain is protected with algorithms and rules defining consensus mechanisms that create decentralized trust and provide social and (crypto)economic incentives to reward honest work (proof-of- $X$ ) and increase reputation of users. Blockchain technologies implement on one hand institutions via coding rules and constraints [67], while on the other they still need institutional governance that must rely on design principles similar to commons [68] such as credible commitment and mutual monitoring [69]. This is especially true here, in our case of I8X platform (described in the next section), where the blockchain technology is of federated or consortium type (the blockchain is governed by the consortium of institutions) so that "the ultimate impact of blockchain in systems of governance and regulation will be felt, not just due its innate characteristics, but its implementation" [70].

The ability of the blockchain— "the trust machine" [71]—-to act as a generator of decentralized trust without any central authority is in our view key to promote cooperation between users, to serve as a indirect reciprocity enabler and reputation builder with perfect memory accessible to anyone. Cooperation is possible even in games with defection (cheating) as the dominant strategy in community of users which frequently interact such as prisoner's dilemma [53,62,72]. Discounting future payment 
in repeated games [53] can maintain cooperation if players are patient and value future earnings enough. Consider an example of a similar game between two players as before with benefit $B=2$ and loss $e=1$. A two-person variation is then given by the following structure shown in Table 2 .

Table 2. Prisoner's dilemma game payoff structure.

\begin{tabular}{ccc}
\hline & $\boldsymbol{C}$ & $\boldsymbol{D}$ \\
\hline$C$ & 1,1 & $-1,2$ \\
$D$ & $2,-1$ & 0,0 \\
\hline
\end{tabular}

A repeated game in which each player adopts a strategy is played in consecutive rounds with payoff $u_{A, k}$ for player $A$ (the row player in Table 2) in round $k$. $A^{\prime}$ s average payoff in infinite game is:

$$
<u_{A}>=(1-\delta) \sum_{k=0}^{\infty} \delta^{k} u_{A, k},
$$

where $\delta=(0,1)$ is a discount factor-a simple measure of player's patience for future payments and trust, or a measure of how the players value reputation [73]. Figure 1 shows a "grim" strategy of the row player $A$ who plays $C$ (cooperation) as long as the other player cooperates. As soon as the column player $B$ defects (plays $D$ ), the player $A$ switches to $D$ in the next and all following rounds.

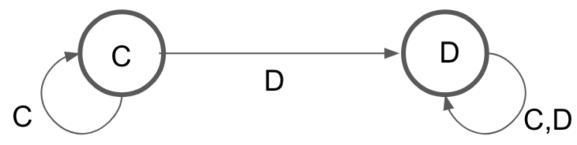

Figure 1. Grim strategy and transitions in repeated game as finite state machine.

With such a strategy and with the payoffs given in Table 1, the players will cooperate if average payoff for cooperation is greater than the average payoff for deviating (defecting):

$$
<u_{C}>\geq<u_{D}>
$$

Average payoff for cooperating is:

$$
<u_{C}>=(1-\delta)\left(1+\delta+\delta^{2}+\ldots\right)=1,
$$

while for defecting after player $A$ defects in the first round we have:

$$
<u_{D}>=(1-\delta)\left(2+0 \delta+0 \delta^{2}+\ldots\right)=2(1-\delta) .
$$

Therefore, a sustained cooperation requires sufficient patience with discount factor $\delta \geq 1 / 2$. In large populations of players with random matching, maintaining cooperation requires larger values of discount factor (Ellison 1994) than in the two player games. If reputations (histories) of players in population of $N+1$ players are known but cannot be communicated or publicly shared in population then cooperation will exist when $\delta \geq 1-1 / 2 N$ [73]. However, in situations where players start with and keep good reputation if they play (using a version of grim strategy as before) $C$ against players with good reputations and play D against players with bad reputations, cooperation is maintained again with $\delta \geq 1 / 2$.

We assert that even in one-shot games describing the situation in scholarly publishing as social dilemma, the blockchain could, as a generator of trust, act as a promoter of cooperation. Let two players $(A, B)$ compete for priority in publishing a paper describing their research [54]. Each can either honestly report their research without additional effort $e=0$ (choose to play $C$ ) or can spend an effort $e>0$ to hype up the paper to make it appear more attractive (choose to play $D$ ). The expected payoff 
for researcher $A$ depends on the reward for publishing $R$, the choice of effort and a function describing the probability to publish that depends on the choices of both researchers:

$$
u_{A}=P\left(e_{A}, e_{B}\right) R-e_{B}
$$

We interpret $P\left(e_{A}, e_{B}\right)$ as the ability of the scientific community as a collective to discern between honest research reporting and hyped up or even fraudulent papers. In publish-or-perish culture, papers that appear more novel and exciting tend to get publishing priority over honest reporting. This is modeled with $P\left(e_{A}=e, e_{B}=0\right)=1$ and $P\left(e_{A}=0, e_{B}=e\right)=0$ for player $A$, describing publication bias found in peer review practice [38]. When the effort of both players is the same, publishing probability equals a coin flip: $P\left(e_{A}=e, e_{B}=e\right)=1 / 2$, reflecting difficulties in recognizing honest research reporting in the current pre-publication review system with small number of reviewers involved in the recommendation process. Choosing $R=4$ and $e=1$, we have the following structure for this game shown in Table 3.

Table 3. Example of prisoner's dilemma game capturing social dilemma in publish-or-perish academic culture.

\begin{tabular}{ccc}
\hline & $\boldsymbol{C}$ & $\boldsymbol{D}$ \\
\hline$C$ & 2,2 & 0,3 \\
$D$ & 3,0 & 1,1 \\
\hline
\end{tabular}

This game portrays a social dilemma since even though mutual cooperation $(C, C)$ offers better payoffs to players, mutual defection - publish-or-perish behavior-is the dominant strategy for both players, and therefore $(D, D)$ is the game's equilibrium.

Blockchain based solutions for publish-or-perish induced problems in scholarly communication must include mechanisms and incentives that promote cooperation between researchers to, in the sense of "publication game", move the game equilibrium towards publish-and-flourish state $(C, C)$. For instance, incentives for the community and mechanisms for lowering the publication bias so that $P\left(e_{A}=e, e_{B}=0\right)=1 / 2$ would lead, ceteris paribus, to the following game structure shown in Table 4 .

Table 4. Example of a coordination game capturing social dilemma in publication game.

\begin{tabular}{ccc}
\hline & $\boldsymbol{C}$ & $\boldsymbol{D}$ \\
\hline$C$ & 2,2 & 0,1 \\
$D$ & 1,0 & 1,1 \\
\hline
\end{tabular}

The game with the structure shown in Table 4 is an example of a coordination game [74] that has two pure equilibria: $(C, C)$ and $(D, D)$ and a mixed equilibrium where both players choose $(D, D)$ with probability $p=0.5$. Obviously, $(C, C)$ publish-and-flourish equilibrium is the preferable outcome for both players, but without communication between players prior to choosing strategy, coordination failures (i.e., choosing $(D, D)$ ) occurs in these types of games [75]. However, using mutual perfect communication prior to playing always leads to $(C, C)$ equilibrium, while in games with noisy communication the selection of strategy $C$ by both players depends on the communication signal [76]. In blockchain supported scholarly communication, such signals are encoded in immutable memory of past events visible to all members of the community.

To promote and maintain users' cooperation and trustworthy publishing and reviewing blockchain supported processes, the key elements—research papers and reviews—of these processes must not be static objects. A continuous (non-static) nature of research papers and reviews refers here to post-publication comments, reviews and curation that take place after the paper has been published, shifting "curation from before to after publication" [77] keeping a research paper 
"alive". Research papers must, therefore, become modifiable entities under persistent review process. A research paper is, in a sense, a contract between author(s) and the community (depending on, for instance, strategic game rules as discussed before). It is an element or outcome of a social contract between science and society under which researchers are expected to communicate their research results to society. A while ago a call for for a new social contract that "will require more open, socially distributed, self-organizing systems of knowledge production that generate their own accountability and audit systems. Under the prevailing contract, science was left to make discoveries and then make them available to society. A new contract will be based upon the joint production of knowledge by society and science" [78] was made. Implementation of a research paper as a smart contract [79] on a blockchain as discussed in the following section might be one possibility on how to imagine knowledge production under new social contract.

\section{The I8X Platform}

In this section we introduce and describe the INFINITCODEX (I8X) (https:/ / dev.infinitcodex. com), scholarly publishing and reviewing blockchain based technology platform (Figure 2). The I8X platform enables and supports entwining the two core processes of scholarly communication-publishing and reviewing-by representing scholarly content as smart contracts and incorporating cryptoeconomical incentives for building reputation in a trustworthy community of users. Here, our intent is to introduce the idea and overview of I8X as a platform supporting various functions of scholarly communication and not to go into implementation details and particular use cases. We are well aware that practical challenges are huge when thinking about disrupting the current models and major players in scholarly communication business. Our goal is to build the platform infrastructure "that enables different providers to offer diverse services-publication of versioned articles from preprints to the final version of record, quality controls before publication, peer review, copy editing, post-publication curation, etc." [77]. There are two processes embedded in the protocol and services layer of the I8X technology stack to support development of applications in the application layer. The infrastructure layer provides compute, storage, database and virtualization support, the protocol layer defines blockchain network participation requirement and rules, method and protocol of consensus, while the services layer contains blockchain services to enable development of applications and connections to other technologies.

\begin{tabular}{|c|c|c|}
\hline Application 1 & Application 2 & Application N \\
\hline \multicolumn{3}{|c|}{ Services } \\
\hline \multicolumn{3}{|c|}{ Protocol layer } \\
\hline \multicolumn{3}{|c|}{ Infrastructure layer } \\
\hline
\end{tabular}

Figure 2. Technology stack of the I8X platform.

The two core processes were designed with three key principles in mind:

- transparency,

- integrity and

- $\quad$ engaging the members of the community. 
Transparency, particularly in blockchain technology context, "is not simply about revealing information or keeping secrets but continually deploying, configuring, and resisting platforms, algorithms, and machine learning protocols that manage visibility" [80]. We agree that it is important to "to differentiate between technical transparency-the ability of a technical solution to make information available and/or accessible-and democratic transparency, an ideal of information availability, accessibility, and actionability meant to serve broader social purposes, including public actor accountability and citizen self-efficacy" [70]. Transparency of the I8X platform is in this sense technological and allows members of the community to overview all blockchain transactions and platform data. On the infrastructure and protocol layer (Figure 2) it is guarded by the consensus mechanisms and network governance. However, we expect that on the application layer level the community will develop solutions on top of the I8X platform that will support "social production technology" [24] of scholarly communication.

How do these key principles of the I8X platform map to scholarly communication functions: registration, certification, legitimization, dissemination, access, preservation, and curation [81]?

Registration is crucial for establishing precedence for scientific claims and attribution of results [81]. Platform functions based on blockchain protocols and governance (consensus rules) provides users/authors with registration function by writing crypotgraphic hash of authors and paper metadata (including the possible link to other preprint or self-archiving servers) on the blockchain following consensus protocol. Immutability of the blockchain record also supports the legitimization function.

The certification function is provided by the reviewing process consisting of comments by the community in the early phases of publication process, and of open peer review process that ends with the decision. Both processes: publication and review are on I8X platform coupled by the community engaged in these scholarly communication functions. This interaction of both processes integrated into I8X platform helps to establish a workflow that "would allow the chaining of specific implementations of the registration, certification, etc. functions into a pathway that could be followed by a unit of communication" [82].

Dissemination and access functions are provided through platform functions and open APIs with different privileges depending on user status, and curation through review process and publication flow. Preservation of digital content is on I8X platform supported by peer-to-peer distributed file systems (such as IPFS-InterPlanetary File System [83]) and in addition by uploading to open preprint servers and self-archiving by the members of the community for redundancy. In a similar way, we can map the I8X key principles to other descriptions of scholarly communication functions such as control and moderation, certification and reputation, incentives [84], or registration, archiving, certification, awareness [85]).

I8X blockchain protocol and developed services such as smart contracts contain incentives (cryptoeconomic as well as social through reputation mechanisms) to stimulate community engagement in all platform activities performed by community members. The functional overview of the interdependence of processes, user interaction and community is shown in Figure 3.

The main function of the I8X platform is to translate user generated content-scholarly communication (research paper, research idea, project proposal, etc.) into curated and reviewed content. The output of the publishing and the review processes are curated reviews, a curated list of trusted reviewers and open data access supported by I8X database of over 150 million metadata points on research papers and high quality index of keywords, authors, organisations and citations. The data on authors, reviews, reputation indices are stored and generated in a decentralized network and accessible to everyone through Open APIs or interaction with smart contracts provided and supported with our I8X technology stack.

There are three types of community members, each with specific roles in I8X platform:

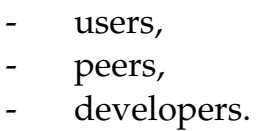


Users are members of the community that interact with I8X platform through the application layer that provides WebUI access and functionality. User actions include claiming existing research articles and other published content, adding new content, reviewing and commenting on the content.

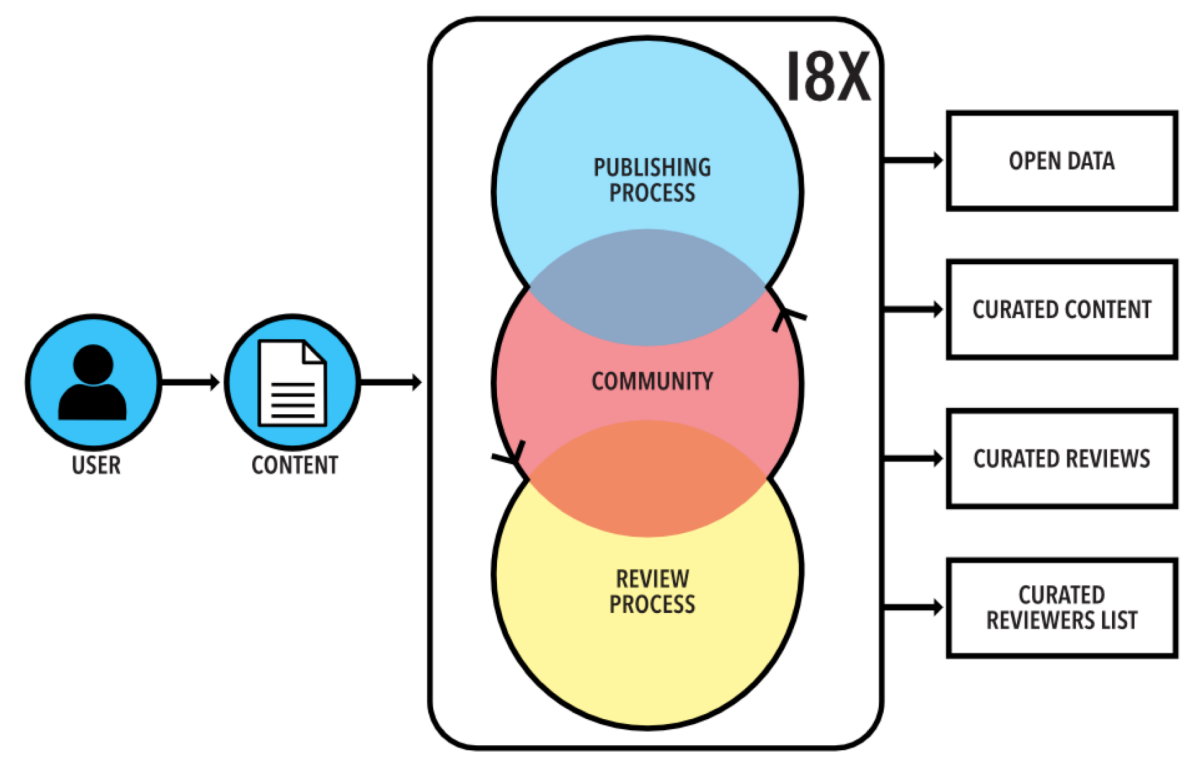

Figure 3. Functional overview of the I8X platform.

Peers are trusted members of the community that form and secure the blockchain network, execute transactions through consensus mechanisms and connect applications with the blockchain.

Developers are community members that use and/or build open APIs, data models and applications using I8X services. I8X applications are decentralized curated content collections. Some examples of applications are: a single research paper, decentralized journal, journal issue or book, decentralized conference proceedings, decentralized research project call.

One of the key parts of developing the I8X platform is the representation and encoding of content (paper, report, abstract, data, code) as smart contract on the blockchain. We take a two-step approach here: first we represent publishing phases of a research paper as a finite state machine, and then encode the process into a smart contract [86].

Consider the process of publishing a research paper as an example. The process is started by the user announcing the intent to publish a paper by submitting the (possibly yet unfinished) content to the platform using one of the applications. The subset of content data (for instance authors, institutions, title, abstract) is hashed and written to the blockchain to secure authorship to authors. The review process (described below) is initiated by the author(s). The outcome of the review process can transition the paper into a published one or return the paper to author(s) for further revisions and development. The community can act also on already published content by raising an objective and proposing the retraction of the published content.

To stimulate good behavior of users, to foster integrity and transparency of the network and to engage community members into actively following publishing and review processes, we integrated several socio-economic incentives and deterrents into the publishing and reviewing processes. As an example, users can use the I8X platform token for depositing in publishing and review processes. By successfully performing certain tasks on the platform such as publishing content, writing reviews and comments, users can earn tokens. The reputation of the user on I8X is a function of number of tokens the users hold at each instant of time. Since the number of tokens reflects the reputation of a user, they can exchange tokens only with I8X platform and not with other users. The internal token economy on I8X for performing functions on the platform serve in this phase as a starting use case for rewarding (and punishing) mechanisms for work done by researchers in scholarly communication 
context (reviewing, commenting, curating, writing, etc.), and for building a community of peers. However, we are well aware that building a trustworthy and sustainable academic reputation system cannot be done in isolation from academic institutions and community.

A finite state machine (FSM) representing the paper has the following states (Figure 4): Active $(A)$, Under review $(U)$, Published $(P)$, and Retracted $(R)$.

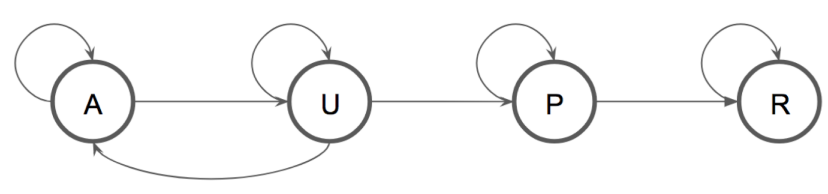

Figure 4. States and transitions in publishing process.

The FSM can change states with the transitions that are initiated by the community members. There are the following transitions in the publishing FSM:

$$
A \Longrightarrow A
$$

In this state transitions are initiated by community comments which preserve the state. Community comments reflect expectations of the community about the research paper. There is no deposit required to submit comments in this state.

$$
A \Longrightarrow U
$$

This transition is initiated by the author when the paper is completed and ready for review. The author deposits $N_{A}>N_{A, M I N}$ tokens and this transaction triggers the start of the review process.

$$
U \Longrightarrow U
$$

While the paper is in this state the review process is in progress. Community members can submit comments about the paper which preserve this state. Commenting in this state works like a prediction market [87-90] where users buy or sell shares for the outcome of the review process (revise or publish).

$$
U \Longrightarrow A
$$

Initiated automatically by the platform as a result of reviewing process (with consensus by the listed reviewers), decision = revise. The author loses the deposited tokens, users that have bought revise shares receive reward.

$$
U \Longrightarrow P
$$

Initiated automatically by the platform as a result of reviewing process (with consensus by the listed reviewers), decision = publish. The author is rewarded for successful publication by $N_{P}>N_{A}$ tokens, and users that have bought publish shares receive reward.

$$
P \Longrightarrow P
$$

In this state users can submit community comments without token deposit and reviews through review process (see below) that can influence reputation of the reviewer and authors.

$$
P \Longrightarrow R
$$


Usually an exceptional transition initiated by community member with "raise an objective" mechanism followed by a dispute/challenge. The outcome is decisioned by consensus of the peers (trusted community members).

$$
R \Longrightarrow R
$$

$R$ is a final state, users can submit comments without depositing tokens.

We base our infrastructure and protocol layers on the family of Hyperledger technologies licensed under the Apache 2.0 license and hosted by The Linux Foundation (https:/ / www.hyperledger.org/). Using these technologies recently proposed to be applied also in the academic publishing field [91], we are building a community blockchain infrastructure and protocols that will support the services of the platform and allow the development of applications.

\section{Conclusions}

Blockchain is a disruptive solution for scholarly communication that "can potentially transform the current socio-technical stasis of moral and market crises associated with academic publishing" [92]. The current scientific publishing system relies on trusted third parties (journals, editorial boards, editors) that provide interactions between authors and reviewers, and there are conflicting incentives and lack of trust among participants. There is definitely a need for an objective, immutable history (or memory) of research results, and for a fair attribution and recognition of authorship that is verified through peer-to-peer consensus mechanisms.

We have presented our implementation of a blockchain based platform for scholarly communication that stands on community driven publishing and reviewing processes and we introduced a research paper as a modifiable entity. Using a strategic game setting, we presented some of the social dilemmas occurring in academic publishing and showed that building the trustworthy scientific community is key for changing the current publish-or-perish culture into a publish-and-flourish one.

Building a trusted peer-to-peer community requires transparency and right incentives for all participants. Various propositions for token-based economy and reward systems in decentralized scholarly publishing have already been advanced [14], including incentives structure in peer review processes based on external cryptocurrency markets [51]. However, what should proper incentives in scholarly communication really achieve? As we have argued here, we believe that whatever the mechanism, the goal must be to establish "a fair game for staking our reputation, so that on questions of interest to funders, we converge as fast as possible to the "right" answer [89].

Author Contributions: All authors contributed equally to this article. The individual contributions of the authors according to the specific tasks in researching and preparing the article are as follows: Conceptualization, A.D. and D.K.; Software, A.D., U.D. (Uroš Droftina), T.K. and U.D. (Urban Duh); Visualization, T.S.K.; Writing-original draft, D.K.; Writing-review \& editing, E.S.D., A.D., U.D. (Uroš Droftina), T.K., U.D. (Urban Duh), T.S.K. and D.K.

Funding: This research received no external funding.

Conflicts of Interest: Authors of this paper are affiliated also with the company Infinitcodex, Ltd. that develops technological solutions for scholarly communications partially based on ideas presented in this paper.

\section{References}

1. Jenkins, H. Convergence Culture: Where Old and New Media Collide; NYU Press: New York, NY, USA, 2006.

2. Duh, A.; Meznaric, S.; Korošak, D. Guerrilla media: Interactive social media. In Media Convergence Handbook-Volume 1; Springer: Berlin, Germany, 2016; pp. 307-324.

3. Vosoughi, S.; Roy, D.; Aral, S. The spread of true and false news online. Science 2018, 359, $1146-1151$. [CrossRef] [PubMed]

4. Duh, A.; Slak Rupnik, M.; Korošak, D. Collective Behavior of Social Bots Is Encoded in Their Temporal Twitter Activity. Big Data 2018, 6, 113-123. [CrossRef] [PubMed] 
5. Lazer, D.M.; Baum, M.A.; Benkler, Y.; Berinsky, A.J.; Greenhill, K.M.; Menczer, F.; Metzger, M.J.; Nyhan, B.; Pennycook, G.; Rothschild, D.; et al. The science of fake news. Science 2018, 359, 1094-1096. [CrossRef]

6. Kirchherr, J. Why We Can'T Trust Academic Journals to Tell the Scientific Truth. Available online: https: / www.theguardian.com/higher-education-network/2017/jun/06/why-we-cant-trust-academicjournals-to-tell-the-scientific-truth (accessed on 14 April 2018).

7. Wüst, K.; Gervais, A. Do you need a Blockchain? In Proceedings of the 2018 Crypto Valley Conference on Blockchain Technology (CVCBT), Zug, Switzerland, 20-22 June 2018.

8. van Rossum, J. Blockchain for Research; Digital Science: London, UK, 2017.

9. Janowicz, K.; Regalia, B.; Hitzler, P.; Mai, G.; Delbecque, S.; Fröhlich, M.; Martinent, P.; Lazarus, T. On the prospects of blockchain and distributed ledger technologies for open science and academic publishing. Semant. Web 2018, 9, 545-555. [CrossRef]

10. Bartling, S.; Friesike, S. Opening Science: The Evolving Guide on How the Internet Is Changing Research, Collaboration and Scholarly Publishing; Springer: Berlin, Germany, 2014.

11. Nakamoto, S. Bitcoin: A Peer-to-Peer Electronic Cash System. 2008. Available online: https://bitcoin.org/ bitcoin.pdf (accessed on 28 May 2019).

12. Buterin, V. A Next-Generation Smart Contract and Decentralized Application Platform. White Paper. 2014. Available online: https://www.weusecoins.com/assets/pdf/library/Ethereum_white_paper_a_ next_generation_smart_contract_and_decentralized_application_platform-vitalik-buterin.pdf (accessed on 28 May 2019).

13. Tapscott, D.; Tapscott, A. Blockchain Revolution: How the Technology behind Bitcoin Is Changing Money, Business, and the World; Penguin: London, UK, 2016.

14. Swan, M. Blockchain: Blueprint for a New Economy; O’Reilly Media, Inc.: Newton, MA, USA, 2015.

15. Brücher, B.L. Science Belongs to No One-And to Everyone. 4open 2018. [CrossRef]

16. Romer, P.M. Mathiness in the theory of economic growth. Am. Econ. Rev. 2015, 105, 89-93. [CrossRef]

17. Ziman, J.M. Reliable Knowledge: An Exploration of the Grounds for Belief in Science; Cambridge University Press: Cambridge, UK, 1978.

18. Hargens, L.L. Scholarly consensus and journal rejection rates. Am. Sociol. Rev. 1988, 53, 139-151. [CrossRef]

19. Cleland, C.E. Prediction and explanation in historical natural science. Br. J. Philos. Sci. 2011, 62, 551-582. [CrossRef]

20. Leonelli, S. Rethinking Reproducibility as a Criterion for Research Quality. In Including a Symposium on Mary Morgan: Curiosity, Imagination, and Surprise; Emerald Publishing Limited: Bingley, UK, 2018; pp. 129-146.

21. Cole, S. The hierarchy of the sciences? Am. J. Sociol. 1983, 89, 111-139. [CrossRef]

22. Nosek, B.A.; Bar-Anan, Y. Scientific utopia: I. Opening scientific communication. Psychol. Inq. 2012, 23, 217-243. [CrossRef]

23. Potts, J.; Hartley, J.; Montgomery, L.; Neylon, C.; Rennie, E. A journal is a club: A new economic model for scholarly publishing. Prometheus 2017, 35, 75-92. [CrossRef]

24. Hartley, J.; Potts, J.; Montgomery, L.; Rennie, E.; Neylon, C. Do we need to move from communication technology to user community? A new economic model of the journal as a club. Learn. Publ. 2019, 32, 27-35. [CrossRef]

25. Brembs, B. Reliable novelty: New should not trump true. PLoS Biol. 2019, 17, e3000117. [CrossRef]

26. Larivière, V.; Haustein, S.; Mongeon, P. The oligopoly of academic publishers in the digital era. PLoS ONE 2015, 10, e0127502. [CrossRef]

27. Bogich, T.; Ballesteros, S.; Berjon, R. On the Marginal Cost of Scholarly Communication. 2016. Available online: https:/ / research.science.ai/bogich2016 (accessed on 28 May 2019).

28. Schmitt, J. Can't Disrupt This: Elsevier and the 25.2 Billion Dollar A Year Academic Publishing Business. 2015. Available online: https://medium.com/@jasonschmitt/can-t-disrupt-this-elsevier-and-the-25-2billion-dollar-a-year-academic-publishing-business-aa3b9618d40a (accessed on 28 May 2019).

29. Bergstrom, T.C. Free labour for costly journals? J. Econ. Perspect. 2001, 15, 183-198. [CrossRef]

30. Papatheodorou, S.I.; Trikalinos, T.A.; Ioannidis, J.P. Inflated numbers of authors over time have not been just due to increasing research complexity. J.Clin. Epidemiol. 2008, 61, 546-551. [CrossRef] [PubMed]

31. Ioannidis, J.P.; Klavans, R.; Boyack, K.W. Thousands of scientists publish a paper every five days. Nature 2018, 561, 167-169. [CrossRef] [PubMed] 
32. Chu, J.S.; Evans, J.A. Too Many Papers? Slowed Canonical Progress in Large Fields of Science. 2018. Available online: https:/ / osf.io/preprints/socarxiv/jk63c/ (accessed on 28 May 2019).

33. Grimes, D.R.; Bauch, C.T.; Ioannidis, J.P. Modelling science trustworthiness under publish or perish pressure. R. Soc. Open Sci. 2018, 5, 171511. [CrossRef] [PubMed]

34. Beall, J. Predatory journals exploit structural weaknesses in scholarly publishing. 4open 2018, 1, 1. [CrossRef]

35. Amaral, O.B. All publishers are predatory-some are bigger than others. Anais da Academia Brasileira de Ciências 2018, 90, 1643-1647. [CrossRef]

36. Smith, R. Peer review: A flawed process at the heart of science and journals. J. R. Soc. Med. 2006, 99, 178-182. [CrossRef]

37. Hatton, L.; Warr, G. Scientific Peer Review: An Ineffective and Unworthy Institution. Available online: https://www.timeshighereducation.com/blog/scientific-peer-review-ineffective-andunworthy-institution (accessed on 14 April 2018).

38. Lee, C.J.; Sugimoto, C.R.; Zhang, G.; Cronin, B. Bias in peer review. J. Am. Soc. Inf. Sci. Technol. 2013, 64, 2-17. [CrossRef]

39. Lee, C.J.; Moher, D. Promote scientific integrity via journal peer review data. Science 2017, 357, $256-257$. [CrossRef]

40. Taichman, D. Misuse of Received Manuscripts by Peer Reviewers: A Cross-Sectional Survey. 2017. Available online: http:/ / peerreviewcongress.org/prc17-0151 (accessed on 28 May 2019).

41. Tomkins, A.; Zhang, M.; Heavlin, W.D. Reviewer bias in single-versus double-blind peer review. Proc. Natl. Acad. Sci. USA 2017, 114, 12708-12713. [CrossRef]

42. Ioannidis, J.P. Why most published research findings are false. PLoS Med. 2005, 2, e124. [CrossRef] [PubMed]

43. Buranyi, S. Is the staggeringly profitable business of scientific publishing bad for science. Guardian 2017, 27, 2017.

44. Smaldino, P.E.; McElreath, R. The natural selection of bad science. R. Soc. Open Sci. 2016, 3, 160384. [CrossRef]

45. Edwards, M.A.; Roy, S. Academic research in the 21st century: Maintaining scientific integrity in a climate of perverse incentives and hypercompetition. Environ. Eng. Sci. 2017, 34, 51-61. [CrossRef]

46. Martinson, B.C. Give researchers a lifetime word limit. Nat. News 2017, 550, 303. [CrossRef]

47. Stephan, P.; Veugelers, R.; Wang, J. Blinkered by bibliometrics. Nature 2017, 544, 411-412. [CrossRef]

48. Higginson, A.D.; Munafò, M.R. Current incentives for scientists lead to underpowered studies with erroneous conclusions. PLoS Biol. 2016, 14, e2000995. [CrossRef]

49. Pepe, A.; Cantiello, M.; Nicholson, J. The arXiv of the future will not look like the arXiv. arXiv 2017, arXiv:1709.07020.

50. Crous, C.J. Could disruptive technologies also reform academia? Web Ecol. 2017, 17, 47-50. [CrossRef]

51. Avital, M. Peer Review: Toward a Blockchain-enabled Market-based Ecosystem. Commun. Assoc. Inf. Syst. 2018, 42, 28. [CrossRef]

52. Maxwell, D.; Speed, C.; Pschetz, L. Story Blocks: Reimagining narrative through the blockchain. Convergence 2017, 23, 79-97. [CrossRef]

53. Fudenberg, D.; Tirole, J. Game theory, 1991. Camb. Mass. 1991, 393, 80.

54. Gall, T.; Maniadis, Z. Evaluating solutions to the problem of false positives. Res. Policy 2019, 48, 506-515. [CrossRef]

55. Leek, J.T.; Taub, M.A.; Pineda, F.J. Cooperation between referees and authors increases peer review accuracy. PLOS ONE 2011, 6, e26895. [CrossRef] [PubMed]

56. Ellison, G. Evolving standards for academic publishing: A q-r theory. J. Polit. Econ. 2002, 110, 994-1034. [CrossRef]

57. Lacetera, N.; Zirulia, L. The economics of scientific misconduct. J. Law Econ. Organ. 2009, 27, 568-603. [CrossRef]

58. Kiri, B.; Lacetera, N.; Zirulia, L. Above a swamp: A theory of high-quality scientific production. Res. Policy 2018, 47, 827-839. [CrossRef]

59. Gall, T.; Ioannidis, J.P.; Maniadis, Z. The credibility crisis in research: Can economics tools help? PLoS Biol. 2017, 15, e2001846. [CrossRef]

60. Stafford, T. Reviews, Reviewers, and Reviewing: The "Tragedy of the Commons" in the Scientific Publication Process. Commun. Assoc. Inf. Syst. 2018, 42, 25. [CrossRef] 
61. Hardin, G. The tragedy of the commons. Science 1968, 162, 1243-1248.

62. Kuhn, S. Prisoner's Dilemma. 2017. Available online: https://plato.stanford.edu/archives/spr2017/ entries/prisoner-dilemma/ (accessed on 28 May 2019).

63. Nowak, M.A.; Sigmund, K. Evolution of indirect reciprocity by image scoring. Nature 1998, $393,573$. [CrossRef]

64. Milinski, M.; Semmann, D.; Krambeck, H.J. Reputation helps solve the 'tragedy of the commons'. Nature 2002, 415, 424. [CrossRef] [PubMed]

65. Van Vugt, M. Averting the tragedy of the commons: Using social psychological science to protect the environment. Curr. Direct. Psychol. Sci. 2009, 18, 169-173. [CrossRef]

66. Ibáñez, L.D.; O’Hara, K.; Simperl, E. On Blockchains and the General Data Protection Regulation. 2018. Available online: https:/ / eprints.soton.ac.uk/id/eprint/422879 (accessed on 28 May 2019).

67. North, D.C. Institutions. J. Econ. Perspect. 1991, 5, 97-112. [CrossRef]

68. Ostrom, E. Governing the Commons; Cambridge University Press; Cambridge , UK, 1990.

69. Walden, J. Past, Present, Future: From Co-Ops to Cryptonetworks. Available online: https://a16z.com/ 2019/03/02/cooperatives-cryptonetworks / (accessed on 8 March 2019).

70. Hofman, D.; Novin, A. Blocked and chained: Blockchain and the problems of transparency. Proc. Assoc. Inf. Sci. Technol. 2018, 55, 171-178. [CrossRef]

71. Economist, T. The promise of the blockchain: The trust machine. Economist 2015, 31. Available online: https:/ / www.economist.com/leaders/2015/10/31/the-trust-machine (accessed on 28 March 2019).

72. Ellison, G. Cooperation in the prisoner's dilemma with anonymous random matching. Rev. Econ. Stud. 1994, 61, 567-588. [CrossRef]

73. Friedman, E.; Resnick, P.; Sami, R. Manipulation-resistant reputation systems. In Algorithmic Game Theory; Nisan, N., Roughgarden, T., Tardos, E., Vazirani, V., Eds.; Cambridge University Press: Cambridge, UK, 2007; Chapter 27, pp. 677-698

74. Cooper, R. Coordination Games; Cambridge University Press: Cambridge, UK, 1999.

75. Cooper, R.; DeJong, D.V.; Forsythe, R.; Ross, T.W. Communication in coordination games. Q. J. Econ. 1992, 107, 739-771. [CrossRef]

76. Carlsson, H.; Van Damme, E. Global games and equilibrium selection. Econometrica 1993, 61, 989-1018. [CrossRef]

77. Stern, B.M.; O'Shea, E.K. A proposal for the future of scientific publishing in the life sciences. PLoS Biol. 2019, 17, e3000116. [CrossRef] [PubMed]

78. Gibbons, M. Science's new social contract with society. Nature 1999, 402, C81. [CrossRef] [PubMed]

79. Szabo, N. Formalizing and securing relationships on public networks. First Monday 1997, 2. [CrossRef]

80. Ananny, M.; Crawford, K. Seeing without knowing: Limitations of the transparency ideal and its application to algorithmic accountability. New Media Soc. 2018, 20,973-989. [CrossRef]

81. Borgman, C.L. Scholarship in the Digital Age: Information, Infrastructure, and the Internet; MIT Press: Cambridge, MA, USA, 2007.

82. Sompel, H.V.; Payette, S.; Erickson, J.; Lagoze, C.; Warner, S. Rethinking scholarly communication: Building the system that scholars deserve. D-Lib Mag. 2004. [CrossRef]

83. Benet, J. Ipfs-content addressed, versioned, p2p file system. arXiv 2014, arXiv:1407.3561.

84. Tennant, J.P. The state of the art in peer review. FEMS Microbiol. Lett. 2018, 365, fny204. [CrossRef]

85. Hummels, H.; Roosendaal, H.E. Trust in scientific publishing. J. Bus. Ethics 2001, 34, 87-100. [CrossRef] [PubMed]

86. Mavridou, A.; Laszka, A. Designing secure Ethereum smart contracts: A finite state machine based approach. arXiv 2017, arXiv:1711.09327.

87. Thicke, M. Prediction Markets for Science: Is the Cure Worse than the Disease? Soc. Epistemol. 2017, 31, 451-467. [CrossRef]

88. Arrow, K.J.; Forsythe, R.; Gorham, M.; Hahn, R.; Hanson, R.; Ledyard, J.O.; Levmore, S.; Litan, R.; Milgrom, P.; Nelson, F.D.; et al. The promise of prediction markets. Science 2008, 320, 877. [CrossRef]

89. Hanson, R. Could Gambling Save Science? Encouraging an Honest Consensus; Taylor \& Francis: Abingdon, UK, 1995.

90. Hanson, R. Logarithmic markets coring rules for modular combinatorial information aggregation. J. Predict. Mark. 2012, 1, 3-15. 
91. Novotny, P.; Zhang, Q.; Hull, R.; Baset, S.; Laredo, J.; Vaculin, R.; Ford, D.L.; Dillenberger, D.N. Permissioned blockchain technologies for academic publishing. Inf. Serv. Use 2018. [CrossRef]

92. Swist, T.; Magee, L. Academic Publishing and its Digital Binds: Beyond the Paywall towards Ethical Executions of Code. Cult. Unbound J. Curr. Cult. Res. 2018, 9, 240-259. [CrossRef]

(C) 2019 by the authors. Licensee MDPI, Basel, Switzerland. This article is an open access article distributed under the terms and conditions of the Creative Commons Attribution (CC BY) license (http:/ / creativecommons.org/licenses/by/4.0/). 